Time-resolved imaging of current-induced domain-wall oscillations

Lars Bocklage $^{1}$, Benjamin Krueger ${ }^{2}$, Rene Eiselt ${ }^{1}$, Markus Bolte ${ }^{1}$, Peter Fischer ${ }^{3}$, and

Guido Meier ${ }^{1}$

${ }^{1}$ Institut fur Angewandte Physik and Zentrum fur Mikrostrukturforschung, Universitat Hamburg, Jungisstrasse 11, 20355 Hamburg

${ }^{2}$ Institut fur Theoretische Physik, Universitat Hamburg, Jungiusstrasse 9, 20355 Hamburg

${ }^{3}$ Center for X-ray Optics, Lawrence Berkeley National Laboratory, One Cyclotron Road, Berkeley, CA 94720 USA

July 2008

This work was supported by the Director, Office of Science, Office of Basic Energy Sciences, of the U.S. Department of Energy under Contract No. DEAC02-05CH11231. 


\title{
Time-resolved imaging of current-induced domain-wall oscillations
}

\author{
Lars Bocklage, ${ }^{1, *}$ Benjamin Krüger, ${ }^{2, \dagger}$ René Eiselt, \\ Markus Bolte, ${ }^{1}$ Peter Fischer, ${ }^{3}$ and Guido Meier ${ }^{1}$ \\ 'Institut für Angewandte Physik und Zentrum für Mikrostrukturforschung, \\ Universität Hamburg, Jungiusstrasse 11, 20355 Hamburg \\ ${ }^{2}$ I. Institut für Theoretische Physik, Universität Hamburg, Jungiusstrasse 9, 20355 Hamburg \\ ${ }^{3}$ Center for X-Ray Optics, Lawrence Berkeley National Laboratory, \\ 1 Cyclotron Road, Berkeley, CA 94720, USA
}

(Dated: July 21,2008 )

\begin{abstract}
Current-induced domain-wall dynamics is investigated via high-resolution soft X-ray transmission microscopy by a stroboscopic pump-and-probe measurement scheme at a temporal resolution of $200 \mathrm{ps.} \mathrm{A}$ $180^{\circ}$ domain wall in a restoring potential of a permalloy microstructure is displaced from its equilibrium position by nanosecond current pulses leading to oscillations with velocities up to $325 \mathrm{~m} / \mathrm{s}$. The motion of the wall is described with an analytical model of a rigid domain wall in a non-harmonic potential allowing to determine the mass of the domain wall. We show that Oersted fields dominate the domain-wall dynamics in our geometry.
\end{abstract}

PACS numbers: $68.37 . Y z, 75.60 . \mathrm{Ch}, 76.50 .+\mathrm{g}, 85.75 .-\mathrm{d}$ 
The quest for novel pathways towards high-density and ultra-fast non-volatile data-storage devices brought magnetic domain walls (DW) and vortices into focus [1-11]. The possibility to manipulate these magnetization configurations by spin-polarized currents is not only scientifically very appealing but also of technological importance in view of novel bit registers $[2,3]$ or logic devices [1]. Vortices and DWs can be moved at high speeds by both field and current excitations, either sinusoidal or pulsed. To control the motion of DWs in nanowires confining potentials, e.g. realized by notches, have been predicted as suitable and locally well defined pinning sites. For small excitations a confining potential can be described by a harmonic oscillator model $[10,11]$. For resonant excitation eigenfrequencies and damping parameters are of interest. Experiments on imaging field-induced vortex or anti-vortex dynamics $[4,5,12,13]$ observe DWs coupled to the motion of a vortex core or an anti-vortex core and other DWs. Direct observation of currentinduced magnetization dynamics has been used very recently to study spin-torque driven vortex gyration [9].

In this Letter we investigate current-induced dynamics of $180^{\circ} \mathrm{DWs}$ in permalloy microstructures by time-resolved X-ray microscopy with $25 \mathrm{~nm}$ spatial and $200 \mathrm{ps}$ time resolution. The spin-polarized current flows directly through the ferromagnetic sample and is accompanied by a magnetic Oersted field. Both can contribute to the excitation of the DW. By analysis of the distinct DW dynamics the dominant contribution can be determined. We image damped DW oscillations during and after the pulse and describe them analytically. Experiments are performed at the full-field soft X-ray transmission microscope (XM-1, beamline 6.1.2), operated by the Center for X-Ray Optics, at the Advanced Light Source in Berkeley, CA. Fresnel zone plates allow for a spatial resolution down to $15 \mathrm{~nm}[14,15]$. A large and element specific magnetic contrast is provided via X-ray magnetic circular dichroism (XMCD) at the $\mathrm{Ni}_{3}$-absorption edge $(852.7 \mathrm{eV})$ [16]. Since the XMCD effect scales with the projection of the magnetization onto the photon propagation direction, in-plane magnetized samples are imaged at a tilt angle of $30^{\circ}$ relative to the surface orientation. A stroboscopic pump-and-probe measurement scheme enables a temporal resolution down to 70 ps [12], which is limited by the inherent time structure of the synchrotron source. Nanosecond current pulses matched to the $3 \mathrm{MHz}$ repetition rate of the X-ray pulses are launched into the ferromagnetic sample to excite the DW dynamics. The current pulses are delayed in steps of $200 \mathrm{ps}$ up to several nanoseconds to obtain a series of temporally resolved X-ray images showing the time evolution of the DW oscillation. The low photon flux of each X-ray pulse requires an integration of each image over billions of events to obtain a sufficient signal-to-noise ratio. 


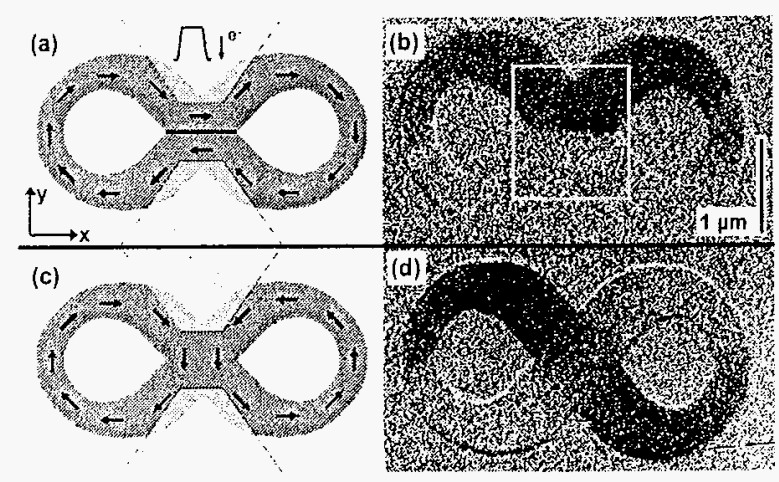

FIG. 1: (Color online) (a) and (c) Scheme of the infinity structure and its magnetizations (arrows). The yellow area illustrates the gold contacts. (b) and (d) Corresponding differential X-ray images showing the static magnetic configuration. The yellow rectangle encloses the section shown in the dynamic images in Fig. 2.

Microstructures of $20 \mathrm{~nm}$ thick permalloy $\left(\mathrm{Ni}_{80} \mathrm{Fe}_{20}\right)$ are prepared on $100 \mathrm{~nm}$ thick $\mathrm{Si}_{3} \mathrm{~N}_{4}$ membranes by electron-beam lithography, thermal evaporation, and lift-off processing. They are contacted by wave guides fabricated via a second lithography step and by DC-magnetron sputtering of $2 \mathrm{~nm} \mathrm{Al}$ and $20 \mathrm{~nm} \mathrm{Au}$.

We investigate "infinity" shaped permalloy microstructures schematically shown in Fig. I(a) and (c). Figure 1(b) and (d) show static differential X-ray images, obtained from an image at remanence which is normalized to an image at saturation along the $\mathrm{x}$-direction. The images at remanence are obtained after saturation along the $\mathrm{x}$-direction. The light and dark contrast indicates that the magnetization remains aligned parallel and antiparallel along the $x$-direction, respectively. Two static magnetic configurations are observed. One configuration is a curling magnetization similar to a global vortex state in a ring [see Fig. 1(a)]. In this case a $180^{\circ} \mathrm{DW}$ is located in the intersection of the infinity. The DW can be seen in the static differential image in Fig. 1(b). The second configuration consists of a clockwise and a counterclockwise curling magnetization in each curvature without a DW at the center of the infinity [see Fig. 1(c) and (d)]. The time-resolved measurements focus on the magnetic configuration with a DW at the center. In contrast to most experiments on single DWs where the domains are magnetized longitudinal to a wire, the investigated wall corresponds to the transversely magnetized configuration. The DW is confined in a strong restoring potential due to pinning of the DW between the pointed holes of the structure. In this way the DW length and energy are minimized. DW oscillations are excited by nanosecond 


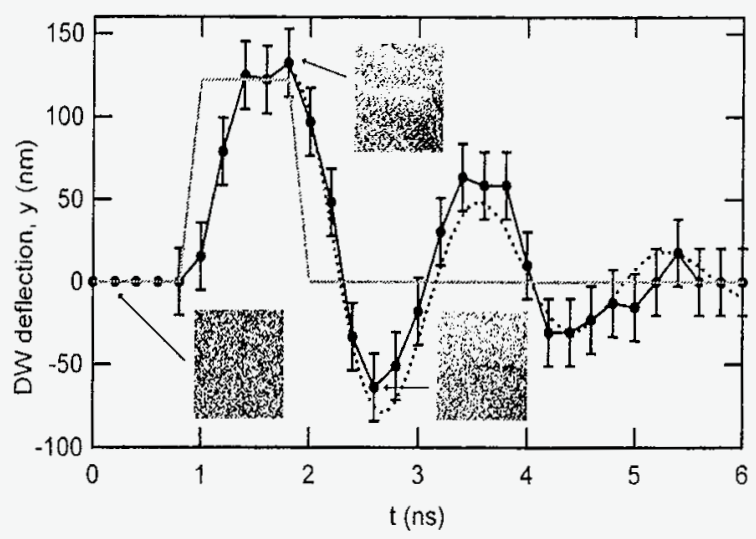

FIG. 2: (Color online) Time evolution of the vertical deffection of the DW (closed circles). The black line is a guide to the eye. Error bars are due to uncertainty in the determination of the deflection. The grey curve depicts the current pulse. The dotted red curve is a fit to the free oscillation using Eq. (1). The images show differential X-ray images of the deflection of the domain wall at 200 ps, 1800 ps, and 2600 ps delay time.

current pulses of a current density of $5 \cdot 10^{11} \mathrm{~A} / \mathrm{m}^{2}$ passing through the ferromagnetic sample [17]. Figure 2 shows the time evolution of the maximal vertical deflection of the DW. Its spatial displacement is indicated by a changing contrast in the insets of Fig. 2 [18]. In principle the contrast is not only given by the spatial displacement of the DW, but also by the change of the out-of-plane component of the DW. The latter contribute less than $5 \%$ to the contrast and is neglected. The dynamic differential $\mathrm{X}$-ray images are obtained from images taken at times $t>0$ which are normalized to the image of the static magnetization at $t=0$ for 30 time steps. As long as the pulse is applied the displacement of the DW persists. Afterwards it starts a free oscillation which is fitted to a simple damped harmonic oscillator

$$
Y(t)=A e^{-\Gamma\left(t-t_{0}\right)} \cos \omega\left(t-t_{0}\right)
$$

Fit parameters are the amplitude $A=125 \mathrm{~nm}$, the damping constant $\Gamma=554 \mathrm{MHz}$, the start time of the free oscillation $t_{0}=1.85 \mathrm{~ns}$, and the free angular frequency $\omega=3.6 \mathrm{GHz}$ which corresponds to the free frequency $f=573 \mathrm{MHz}$. The eigenfrequency of the system $\omega_{0}=\sqrt{\omega^{2}+\Gamma^{2}}$ is $3.64 \mathrm{GHz}\left(f_{0}=578 \mathrm{MHz}\right)$ and the damping time $\tau_{d}=1 /(2 \Gamma)$ is $0.9 \mathrm{~ns}$. These parameters describe the harmonic potential.

Figure 3 shows time scans of the deflection of the DW due to current pulses of different pulse lengths [19]. During the pulse an oscillation of the DW around the deflected position is observed. 


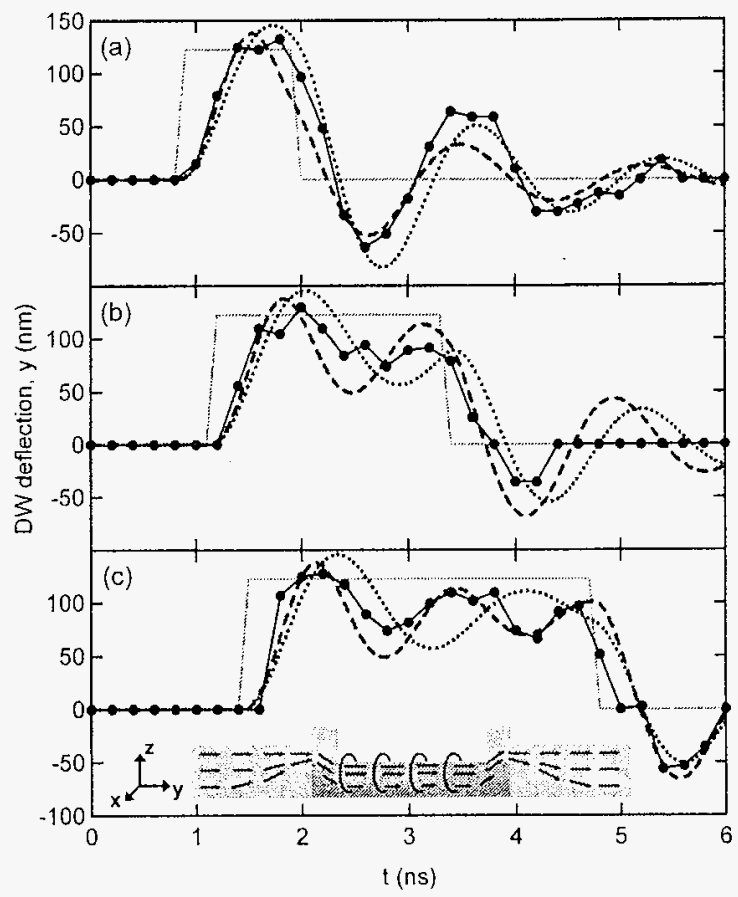

FIG. 3: (Color online) Time evolution of the vertical deflection of the DW (closed circles) for current pulses with a width of (a) $1.1 \mathrm{~ns}$, (b) $2.2 \mathrm{~ns}$, and (c) $3.3 \mathrm{~ns}$. Black lines are guides to the eye. Errors are the same as in Fig. 2. The grey curves depict the current pulses. Theoretical curves are calculated with Eq. (5) for the harmonic potential (dotted red) and the non-harmonic potential (dashed blue). The inset shows a scheme of the contacted permalloy structure. The arrows illustrate the current through the sample. Its Oersted field is indicated in red.

Afterwards the free oscillation starts.

The derivatives of both the measured and the fitted data for a pulse length of $1.1 \mathrm{~ns}$ are shown in Fig. 4(a). The maximum measured velocity of the DW is $325 \mathrm{~m} / \mathrm{s}$. This is the velocity of the DW driven by the demagnetization and exchange field. We also varied the amplitude of the current pulses. Fig. 4(b) shows the maximal measured deflection in a time scan for different current densities. The pulse width is 2.2 ns. We observe a linear dependence of the deflection on the current density and deduce from a linear regression a threshold current density of $2.7 \cdot 10^{11} \mathrm{~A} / \mathrm{m}^{2}$. Fig. 4(c) shows the vertical deffection of the DW due to static magnetic fields. The DW shows the expected behavior and changes the direction of the deflection if the sign of the magnetic field is changed [20]. 

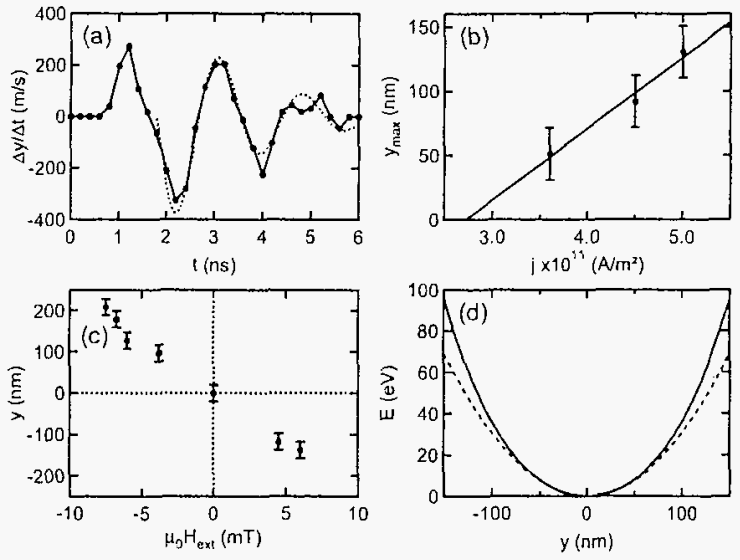

FIG. 4: (a) Derivatives of the measured data (closed circles) and the fit (dotted red curve) shown in Fig. 2. The black line is a guide to the eye. (b) Dependence of the maximal vertical deflection on the current density. Plotted is the maximal deflection in time scans for a pulse length of $2.2 \mathrm{~ns}$. The black line is a linear fit. (c) Vertical deflection of the DW due to a static magnetic field in $\mathrm{x}$-direction for the magnetic configuration of Fig. 1(a) and (b). (d) Potential of the DW for the harmonic (dashed line) and the non-harmonic confinement (solid line) as deduced from the experiments.

Since every current is accompanied by an Oersted field two excitation processes of the DW compete: spin-angular momentum-transfer and magnetic fields. A non-vanishing Oersted field in the plane of the permalloy is generated by an inhomogeneous current flow due to the position of the contact pads on top of the structure and the ratio of the specific resistivity of Au to permalloy of 20 for our samples (see inset of Fig. 3). To distinguish between the contributions their distinct effects on the DW are now discussed. As depicted in Fig. 1(a) the electrons flow from the upper to the lower contact. The experimentally observed initial motion of the DW is upwards (see Fig. 2). However, in case of spin-torque excitation the expected initial motion of the DW is a displacement in the direction of the electron flow [21]. Furthermore the initial motion of the DW is inverted in the case of the inverted magnetic configuration where the magnetization runs counterclockwise around the structure (not shown) [22]. Thus spin torque is not the main source of the excitation in the present experiment and we conclude that the observed displacement of the DW is due to Oersted fields. The linear dependence of the DW deflection on the current density (see Fig. 4(b)) originates from the linear dependence of the Oersted field on the current. The distinction between spin-torque and Oersted field contributions to the motion of the magnetization is 
so far only possible with temporally and spatially resolved experiments like X-ray microscopy [9]. The contribution of Oersted fields to the dynamics of the magnetization is a general property of contacted ferromagnetic structures where electric contacts are close to the investigated magnetic configuration, like in experiments on vortex-core motion or vortex-core switching in squares and disks $[8,9]$. In experiments on DW motion in long wires $[3,6,7]$ the issue is not so prominent since a sufficiently long distance between the magnetic structure and the contacts exists allowing the current density to equilibrate.

To analytically describe the DW oscillations during and after the pulse, we assume that the motion is mainly caused by an Oersted field. The dynamics of the magnetization $\vec{M}$ can then be described by the Landau-Lifshitz-Gilbert (LLG) equation

$$
\frac{d \vec{M}}{d t}=-\gamma \vec{M} \times \vec{H}_{\mathrm{cff}}+\frac{a}{M_{S}} \vec{M} \times \frac{d \vec{M}}{d t}
$$

Here $\vec{H}_{\text {eff }}$ is the effective magnetic field, $\gamma=g \mu_{0} \mu_{B} / \hbar$ is the gyromagnetic ratio, $\alpha$ the Gilbert damping parameter, and $M_{S}$ the saturation magnetization. We further assume a rigid wall [23], for the analytical description. The equations of motion for the DW, derived from the LLG equation, then read

$$
\dot{Y}=-\frac{2 \lambda \gamma_{\gamma}^{\prime} K_{\perp}}{\mu_{0} M_{S}} \phi-\lambda \gamma^{\prime} \alpha H(Y)
$$

and

$$
\dot{\phi}=\gamma^{\prime} H\left(Y^{-}\right)-\frac{2 \gamma^{\prime} \alpha K_{\perp}}{\mu_{0} M_{S}} \phi
$$

with $\gamma^{\prime}=\gamma /\left(1+\alpha^{2}\right)[10]$. The DW is described as a rigid particle by the position of the center of the wall $Y$ and the angle of the rotation of the wall out of the plane $\phi$. The anisotropy constant for the magnetization pointing in the y-direction is $K$. For the magnetization pointing out-of-plane the anisotropy is $K+K_{\perp}$. The width of the DW is $\lambda=\sqrt{A / K}$ with the exchange constant $A$. The magnetic field $H$ consists of the external field $H_{\text {ext }}$ and the pinning field $H_{\text {pin }}$. The external field points in-plane perpendicular to the current, i.e. in $x$-direction. From Eq. (3) and (4) the relation

$$
\ddot{Y}=-\frac{\lambda \gamma H_{\mathrm{ext}}+\alpha \dot{Y}}{\alpha \tau_{d}}-\lambda \gamma^{\prime} \alpha \dot{H}_{\mathrm{cxt}}-\frac{1}{m} \frac{d E}{d Y}
$$

for the position of the DW is derived, with the damping time $\tau_{d}=\mu_{0} M_{S} /\left(2 \gamma^{\prime} \alpha K_{\perp}\right)$ and the DW mass $m$. Here, the pinning field is expressed by the pinning potential $E$. The time derivative of the pinning field is small and can be neglected. In the absence of a temporally varying external field, Eq. (5) describes a damped oscillation. Note, that the dynamics is determined by the width of 
the wall. With the pinning potential $E$ from the model of a damped harmonic oscillator [Eq. (1)] we obtain the entire temporal evolution of the DW. By a time integration of Eq. (5) the velocity and the position of the DW can be calculated. The time integration is done numerically using the explicit Euler method with $\alpha=0.01$ of permalloy. A harmonic pinning potential $E=\frac{1}{2} m \omega^{2} Y^{2}$ does not describe the dynamics very well. This is shown by the best fits plotted as dotted red lines in Fig. 3.

The large deflection of the DW leads to deviations from a harmonic potential. Adding a term of fourth order to the potential, meaning a stronger force on the wall, the potential reads $E=$ $\frac{m \omega^{2}}{2} Y^{2}+\frac{m k}{4} Y^{4}$. With $\mu_{0} H_{\mathrm{ext}} \cdot \lambda=-7.5 \cdot 10^{-11} \mathrm{Tm}$ and $k=4.5 \cdot 10^{32} \mathrm{~s}^{-2} \mathrm{~m}^{-2}$ the theory fits the measurements very well. The non-harmonic potential changes the frequency at large deffections as can be seen in Fig. 3. Fig. 4(d) shows the deduced harmonic and non-harmonic potential. One can see that the higher order terms are negligible for small deflections. The extracted harmonic potential obtained from the data in Fig. 2 is fitted to such small deflections, therefore the parameters of the harmonic potential hold true in the analysis [24].

The DW width $\lambda=(23 \pm 9) \mathrm{nm}$ is determined from Eq. (5) by setting all time derivatives zero and using the measured deflection of the DW of $(97 \pm 20) \mathrm{nm}$ at an applied constant field of $(-3.8 \pm 0.8) \mathrm{mT}$ (see Fig. 4(c)). From $\mu_{0} H_{\mathrm{ext}} \cdot \lambda$ an Oersted field of $\mu_{0} H_{\mathrm{ext}}=(-3.3 \pm 1.2) \mathrm{mT}$ is calculated. This value is comparable to the static fields needed to deflect the DW $130 \mathrm{~nm}$. One should keep in mind that we assume a rigid Neel wall. From the images one can see that the DW rather tends to bend (see Fig. 2). Anyhow the model describes the DW motion very well. Numerical calculations of the Oersted field on similar structures have shown that fields of a few Millitesla can be generated [9].

For our theoretical description of the DW motion it is important to verify that the angle $\phi$ is small as the equations of motion have been linearized in Ref. [10]. With the maximum experimental velocity of $-325 \mathrm{~m} / \mathrm{s}$ (the Oersted field is zero at this time), Eq. (3) leads to $\phi=(7.37 \pm 0.05)^{\circ}$ which is in the linear regime.

The characteristic parameters of the harmonic oscillator depend on the micromagnetic material parameters of permalloy. The damping parameter is given by

$$
\Gamma=\frac{1}{2 \tau_{d}}=\frac{\alpha \gamma^{\prime} K_{\perp}}{\mu_{0} M_{S}}
$$

To estimate the accuracy of $\alpha=0.01$ we calculate $K_{\perp}$ with Eq. (6) and find that the value for $K_{\perp}$ of $2.7 \cdot 10^{5} \mathrm{~J} / \mathrm{m}^{3}$ using $M_{S}=8.6^{6} \cdot 10^{5} \mathrm{~A} / \mathrm{m}$ is reasonable for our geometry. In Ref. [10] the DW 
mass

$$
m=\frac{S \mu_{0}^{2}}{\lambda \gamma^{\prime 2} K_{-}^{2}}=\frac{2 \alpha S \mu_{0} M_{s} \tau_{d}}{\lambda \gamma^{\prime}}
$$

was calculated. With the known values for the cross section $S=2 \cdot 10^{-14} \mathrm{~m}^{2}$ and the damping time $\tau_{d}=0.9 \mathrm{~ns}$ this yields a mass of $m=(7.6 \pm 2.8) \cdot 10^{-23} \mathrm{~kg}$. This DW mass is comparable to previous results [25], although our DW structure differs as discussed.

In summary we have imaged the dynamics of a DW due to nanosecond current pulses with high temporal and spatial resolution using X-ray microscopy. The results allow to identify the current's Oersted field as the main source of excitation of the magnetization although the current flows directly through the sample. From the experiment the potential of the confined DW is extracted. An analytical model which is capable to precisely describe the field-driven dynamics of the DW is presented. A potential with higher than harmonic terms determines the motion of the DW.

We thank Danicla Pfannkuche, Toru Matsuyama, and Ulrich Merkt for fruitful discussions as well as Sandra Motl for assistance with the analysis of the X-ray images. Financial support by the Deutsche Forschungsgemeinschaft via the SFB 668 and via the Graduiertenkolleg 1286 is gratefully acknowledged. Operation of the soft X-ray microscope is funded by the Director, Office of Science, Office of Basic Energy Sciences, Materials Sciences and Engineering Division, of the U.S. Department of Energy.

* Electronic address: 1bocklag@physnet.uni-hamburg.de

$\dagger$ Electronic address: bkrueger@physnet.uni-hamburg.de

[1] D. A. Allwood et al., Science 309, 1688 (2005).

[2] S. S. P. Parkin, Science 320, 190 (2008).

[3] M. Hayashi et al., Science 320, 209 (2008).

[4] S.-B. Choe et al., Science 304, 420 (2004).

[5] B. Van Waeyenberge et al., Nature 444, 461 (2006).

[6] L. Thomas et al., Nature 443, 197 (2006).

[7] G. Meier et al., Phys. Rev. Lett. 98, 187202 (2007).

[8] K. Yamada et al., Nat. Mater. 6, 269 (2007).

[9] M. Bolte et al., Phys. Rev. Lett. 100, 176601 (2008).

[10] B. Krüger et al., Phys. Rev. B 75, 054421 (2007). 
[11] B. Krüger et al., Phys. Rev. B 76, 224426 (2007).

[12] A. Puzic et al., J. Appl. Phys. 97, 10E704 (2005).

[13] K. Kuepper et al., Phys. Rev. Lett. 99, 167202 (2007).

[14] W. Chao et al., Nature 435, 1210 (2005).

[15] D.-H. Kim et al., J. Appl. Phys. 99, 08H303 (2006).

[16] C. Chen et al., Phys. Rev. B 42, 7262 (1990).

[17] The experimental high frequency setup is accounted by $-3 \mathrm{~dB}$ in the calculations of the current density.

[18] See also moviel.avi in the online supporting material.

[19] See also moviel.avi, movie2.avi, and movie3.avi in the online supporting material.

[20] Sending pulses through the sample with applied static fields generates an oscillation around the new equilibrium position.

[21] S. Zhang and Z. Li, Phys. Rev. Lett. 93, 127204 (2004).

[22] In a reversed magnetic configuration the DW initially moves in the inverse direction when the Oersted field remains the same. It is similar to change the field direction on an unchanged magnetic configuration, compare Fig. 4(c).

[23] W. Döring, Z. Naturforsch. 3a, 373 (1948).

[24] Only for the first two data points of the fit in Fig. 2 the deflection exceeds $64 \mathrm{~nm}$. At $64 \mathrm{~nm}$ the difference of the two potentials is $7 \%$.

[25] E. Saitoh et al, Nature 432, 203 (2004). 